\title{
APLICAÇÃO DO MÉTODO SIMPLE AGGREGATION OF PREFERENCES EXPRESSED BY ORDINAL VECTORS - MULTI DECISION MAKERS (SAPEVO-M) NA SELEÇÃO DE FORNECEDORES DE CAIXA DE PAPELÃO
}

\author{
Guilherme Castro Xavier da Silva \\ Universidade do Grande Rio (UNIGRANRIO) \\ Rua Professor José de Souza Herdy, 1160, Duque de Caxias/RJ \\ guilhermexavier@unigranrio.br
}

Marcos dos Santos

Centro de Análises de Sistemas Navais (CASNAV) / Instituto Militar de Engenharia (IME) Praça Barão de Ladário, Ilha das Cobras, Rua da Ponte, Ed.23, Centro, Rio de Janeiro/RJ marcosdossantos doutorado uff@yahoo.com.br

Luiz Frederico Horácio de Souza de Barros Teixeira

Centro de Análises de Sistemas Navais (CASNAV)/ Universidade Federal Fluminense (UFF) Praça Barão de Ladário, Ilha das Cobras, Rua da Ponte, Ed. 23, Centro, Rio de Janeiro/RJ frederico.horacio@gmail.com

Carlos Francisco Simões Gomes

Universidade Federal Fluminense (UFF)

Rua Passo da Pátria, 156 - 209, São Domingos, Niterói - RJ

cfsg1@bol.com.br

Angélica Rodrigues de Lima

Centro de Tecnologia da Indústria Química e Têxtil (SENAI CETIQT)

Rua Magalhães Castro, $n^{\circ}$ 174, Riachuelo, Rio de Janeiro/RJ

angeliquinha.lima@gmail.com

\section{RESUMO}

O Centro de distribuição da empresa Alfa, localizado em Duque de Caxias, é responsável por atender diariamente as entregas de todas as regiões do estado do Rio de Janeiro, o que gera uma grande operação de distribuição diariamente, que é realizada de forma muito eficiente. Todos esses pedidos, após faturados, são separados, identificados e alocados em caixas de papelão, que seguem um padrão de forma e tamanho, para melhor conferência e alocação dos produtos. Isso faz com que a empresa necessite comprar caixas de papelão para que se possa atender toda a demanda de pedidos, porém não é qualquer caixa que atende as necessidades da empresa, para que a operação funcione de forma eficiente e eficaz, as caixas devem seguir uma pré determinação elaborada pela empresa. Em meio a pesquisas realizadas no mercado, foram elencados três fornecedores que atendiam as exigências e critérios da empresa, a fim de auxiliar a tomada de decisão, para se definir um excelente fornecedor, foi aplicado o método multicritério SAPEVO-M ao estudo, para que se possa encontrar a melhor opção de fornecedor de caixas de papelão para empresa.

Palavra-chave: Caixas de papelão; Centro de distribuição; Método SAPEVO-M. 


\begin{abstract}
Write your abstract here. The distribution center of the Alfa company, located in Duque de Caxias, is responsible for delivering daily deliveries to all regions of the state of Rio de Janeiro, which generates a large daily distribution operation, which is carried out very efficiently. All of these orders, after invoiced, are separated, identified and allocated in cardboard boxes, which follow a pattern of shape and size, for better conference and product allocation. This means that the company needs to buy cardboard boxes so that it can meet all the demand for orders, but it is not any box that meets the needs of the company, so that the operation works efficiently and effectively, the boxes must follow a pre-determination prepared by the company. In the midst of market research, three suppliers were listed that met the requirements and criteria of the company, in order to help decision making, in order to define an excellent supplier, the SAPEVO-M multicriteria method was applied to the study, so that you can find the best option from carton supplier to company.
\end{abstract}

Keywords: Cardboard boxes; Distribution center; SAPEVO-M Method.

\title{
1. INTRODUÇÃO
}

A empresa com o pseudônimo “Alfa” é uma indústria de cigarros brasileira, que atua diretamente em toda as áreas de seu negócio, desde a produção até a entrega do produto final aos clientes. Atualmente essa Companhia possui seis Centrais Integradas de Distribuição (CID), espalhadas pelo país e como complemento a sua rede de abastecimento, ainda possui 31 Centrais Operacionais de Distribuição (COD) localizadas nas grandes cidades e capitais, além de postos de abastecimento (PA) no interior do país. Ao todo a Companhia atende cerca de 300 mil pontos de venda em mais de 5.000 mil municípios brasileiros, o que representam quase $95 \%$ do total do país.

Os centros de distribuição, espalhados pelo país, recebem das fábricas os produtos, e os armazenam, a fim de processar os pedidos, montá-los e distribui-los, pelas regiões em que o centro atende. Para que o processo seja feito, após o faturamento dos pedidos, é gerado um valor bruto da quantidade necessária de cada produto para a montagem dos pedidos. Essa quantidade bruta é retirada dos estoques e disponibilizada para as esteiras de montagem de pedidos. Cada colaborador é responsável por um escopo de produto acomodado na região de trabalho; logo, ao ver a nota com a descrição do produto solicitado, deve-se identificar a quantidade solicitada de cada produto, e acomodá-lo na caixa de papelão correspondente a nota. Depois de efetuar sua atividade, coloca-se a nota novamente na caixa de papelão, e a empurra pela esteira, para que o próximo colaborador, faça o mesmo processo, com os devidos produtos. Segue-se o mesmo processo até o fim da esteira, onde é realizada uma conferência das notas e do total de produtos e, em seguida, as caixas são devidamente identificadas e despachadas para o carro de entrega.

Os produtos são diversos, porém sua estrutura e sua forma são padronizadas, de modo que a sua acomodação nas caixas de papelão segue um padrão, a fim de facilitar a contagem e conferência desses ao fim da manufatura. Da mesma maneira, as caixas de papelão também devem seguir um padrão de tamanho para proporcionar melhor acomodação dos produtos e facilitar o processo de montagem e conferência dos mesmos. As caixas de papelão tornam-se de extrema importância para esse processo, visto que, uma vez sem a quantidade necessária delas, a operação é forçada a parar, por não ter onde acomodar os produtos e fazer a montagem dos pedidos. 


\section{DESCRIÇÃO DO PROBLEMA}

\subsection{Montagem dos Pedidos}

Para todo o processo feito após o faturamento dos pedidos é necessário haver caixas de papelão para acomodação dos produtos. Uma vez que as demandas são geradas, uma grande quantidade de produtos é retirada do estoque para atender a demanda diária. Consequentemente, caixas fechadas dos produtos são trazidas para o processo de montagem de pedidos. Estas caixas chegam prontas das fábricas dos produtos e seguem um padrão para a acomodação dos mesmos. Assim, as próprias caixas também são utilizadas na montagem de pedidos, porém apenas essas caixas originais dos produtos não são suficientes para atender toda a demanda de pedidos gerados. Isto se deve ao fato de que são diferentes tipos de pedidos, no qual são contidas quantidades e produtos diversos. Portanto, é necessário que seja realizada a compra de mais caixas de papelão para que o processo possa ocorrer de forma ininterrupta.

As caixas de papelão tornam-se um elemento fundamental para a realização das atividades de produção pois sem as mesmas não é possível conduzir o processo de montagem e realizar as entregas. Em alguns casos muito extremos, onde a demanda é grande, pode ocorrer a paralização na operação pela falta de caixas. A escassez de caixas, ou a utilização de caixas fora dos padrões ou de má qualidade, atrasam o processo, o que por sua vez acabam afetando o seu lead time. Isto afeta diretamente nos indicadores de produtividade das esteiras, criando outro problema para empresa, uma vez que todo o processo se atrasa, os colaboradores não conseguem terminar as atividades no tempo padrão de serviço, gerando a necessidade de realizar hora extra.

\subsection{Caixas Utilizadas no Processo}

O Centro de distribuição do Rio de Janeiro sofre com a falta de caixas. Como uma forma de aliviar essa escassez, solicitou-se através de contatos e negociações com as fabricas, um envio extra de caixas vazias para o Centro de distribuição. Contudo, esses envios não são regulares e os mesmo não atendem toda a demanda, tornando inevitável a compra de caixas de papelão. Entretanto, como as caixas, seguem um padrão de tamanho e formatos, a empresa busca fornecedores que possam atender as exigências da melhor maneira possível. As caixas além do formato e tamanho, devem conter também uma boa qualidade, a fim de proporcionar ao cliente uma entrega satisfatória, com a confiabilidade de que seu produto está bem acomodado e em perfeito estado. Além disso, por muitas vezes a empresa precisa de um tempo de entrega rápido e eficaz a fim de não sofrer paralizações forcadas pela falta de caixas de papelão, logo, um tempo de entrega rápido e confiável, é um diferencial para a busca de fornecedores.

Face ao exposto, o presente estudo busca analisar qual será o melhor fornecedor de caixas de papelão, uma vez que este deve fornecer as caixas dentro de um padrão de tamanho e formato pré-definidos. Além disso, espera-se que o fornecedor entregue uma caixa de papelão de alta qualidade para garantir a melhor entrega ao consumidor e agilizar o processo possibilitando uma entrega confiável, a fim de suprir uma demanda extra de forma rápida. O método SAPEVO-M, será utilizado para se obter uma conclusão bem fundamentada dos critérios, a fim de auxiliar a tomada de decisão.

A fim de facilitar a compreensão e delimitação do escopo do problema foi construído um mapa mental apresentado na Figura 1 a seguir. 


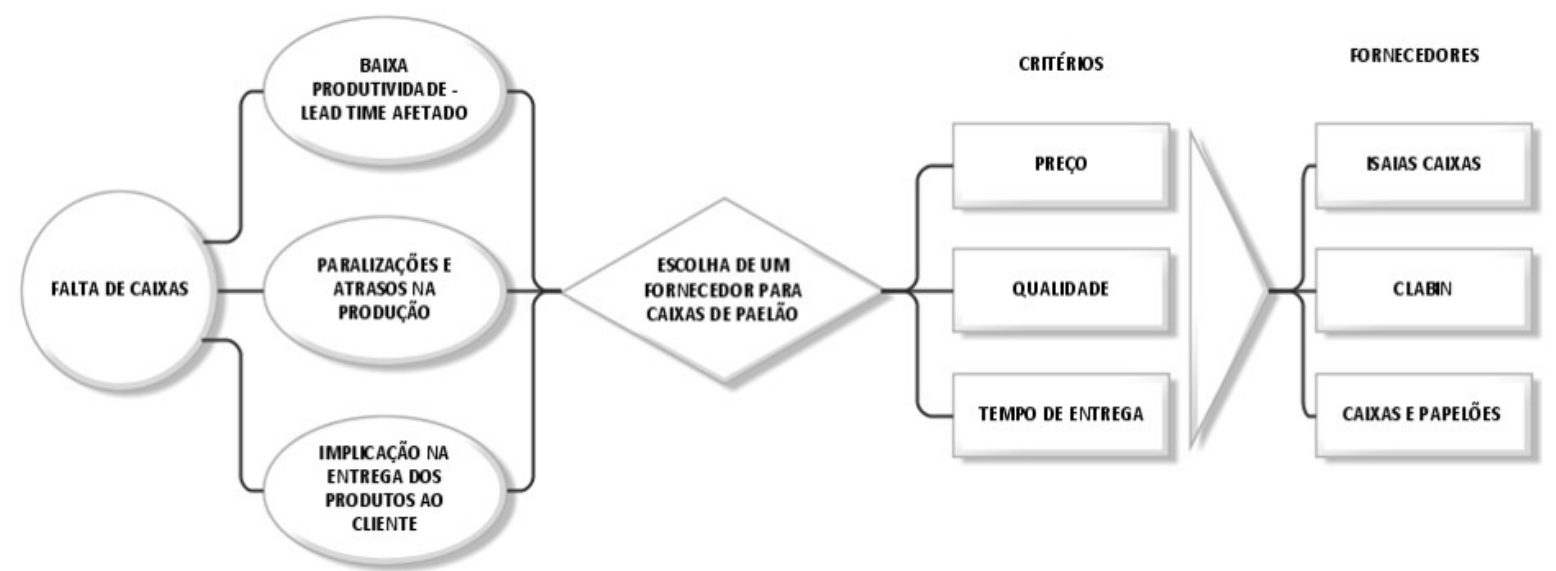

FIGURA 1 - Problema decisório da seleção de fornecedores de caixas de papelão. Fonte: Autores (2019).

\section{FUNDAMENTAÇÃO TEÓRICA}

\subsection{COMPeTITIVIDAde DE Mercado}

No atual cenário mundial, globalizado, onde a competitividade de mercado está cada vez mais acirrada e cada vez mais pessoas e grupos tem fácil acesso a todo tipo informação, as empresas estão sendo obrigadas a inovar seus métodos e serviços a fim de se manterem competitivas no mercado (CARVALHO, 2017).

Mas durante muitos anos a realidade já foi outra. Em tempos passados onde não havia fácil acesso às informações, a tecnologia era muito cara e restrita, os processos eram lentos, tudo era mais demorado e previsível. Desta forma, não havia muita flexibilidade, e isso fez com que as grandes e ricas empresas se consolidassem no mercado como grandes impérios. Nessa fase onde tudo era muito igual, e tudo demorava muito para mudar, o indivíduo era obrigado a se adequar as exigências desses grandes grupos (MOHR et al., 2011).

Com o passar do tempo e o grande aumento da tecnologia, o cenário mudou e tudo se tornou mais transparente, complexo, imprevisível e mais rápido. Consequentemente, isto fez com que as grandes instituições, antes inabaláveis, começassem a se reinventar e buscar inovar seus métodos, agora se adequando as exigências do mercado, que se encontra cada vez mais instável e fora de controle (MOHR et al., 2011).

Dentro desse contexto, pode-se imaginar o crescimento da competitividade entre os mais diversos mercados. E as empresas neste cenário buscam meios de se manterem competitivas, seja, inovando e se adaptando as novas exigências, seja reduzindo ao máximo seus custos operacionais. Um conceito antes não muito conhecido começa a surgir em meio ao cenário produtivo: o conceito de Qualidade. As empresas, de uma forma geral, passam a buscar agregar valor a seus produtos, seus processos, sistemas, de maneira a melhorar algum aspecto, que gere um diferencial a elas no mercado globalizado (ANDREOLI; BASTOS, 2017).

\subsection{Métodos de Apoio Multicritério À DecisÃo (AMD)}

Com o grande aumento da tecnologia, as informações tornaram-se mais acessíveis a todos. Hoje, com o grande número de informações que chegam a todo momento e se renovam a cada dia, o indivíduo se vê obrigado a utiliza-las da melhor maneira possível, porém se torna muito complexo administrar todas as informações geradas, logo, a fim de auxiliar o processo decisório, surgiu os métodos multicritérios, auxiliando os tomadores de decisão, a fim de alcançar uma maior assertividade na tomada de decisão (BRIOZO, MUSETTI, 2015). 
Segundo Costa et al, (2013) em um ambiente organizacional é normal que diariamente pessoas tenham que tomar decisões voltadas ao trabalho, de forma contínua. Porém, em alguns casos, as decisões não são tão simples. Em ambientes complexos, as dificuldades para uma tomada de decisão são cada vez maiores, devido às muitas variáveis e informações envolvidas no processo e na organização em questão. Muitos podem ser os aspectos que diferenciam e, até mesmo, dificultam a tomada de decisão, como questões culturais, monetárias, pessoais, legislativos, entre muitos outros. As empresas, seja qual for seu setor de atuação necessita que decisões sejam tomadas a todo momento; para tal, elas podem empregar os vários métodos que se aplicam a múltiplos critérios, utilizando diferente abordagens para conseguir alcançar uma melhor decisão. Com isso, as empresas podem conseguir uma metodologia eficaz de enfrentar as dificuldades do mercado atual.

Gomes e Gomes (2019) definem os métodos de apoio multicritério à decisão (AMD) como técnicas de assessoramento ao agente decisor (quer seja indivíduo, grupo de indivíduos, comitê de especialistas ou lideranças) para a tomada de decisões sobre problemas complexos, selecionado, avaliando ou ordenando alternativas mediante diferentes pontos de vista e dentro de critérios pré-definidos.

Para Wernke e Bornia (2001), os métodos multicritérios agregam grande valor às informações, na medida em que, além de facilitarem a abordagem de problemas complexos, de difícil tratativa pelos procedimentos intuitivos usuais, também trazem ao processo de decisão uma clareza e transparência. Isto é, os métodos AMD são capazes de reconhecer uma necessidade em uma abordagem mais abrangente, através da utilização de múltiplos fatores, que façam parte dos vários e diversos aspectos da empresa.

Como a sociedade tende a se tornar cada vez mais complexa, há a necessidade de tornar os objetivos cada vez mais bem estabelecidos, de maneira que as pessoas tendem a tomar diferentes tipos de decisões, o processo de tomada de decisão torna-se cada vez mais conflitante, uma vez que diversas opiniões e informações, muitas vezes não agregam nada ao seu verdadeiro objetivo, e isso implica a ao decisor, tomar decisões com múltiplos critérios (REIS e LÖBLER, 2012).

Para o auxílio à tomada de decisão é importante definir bem seus critérios, sendo necessário que o tomador de decisão (Decision Maker - DM) conheça sua real necessidade. Assim, espera-se que o DM possa elencar critérios que eliminem possíveis opções que não acrescentariam em nada a solução do problema, Os critérios podem ser bem definidos, porém entre eles pode haver um com mais importância do que o outro; logo é fundamental a definição de pesos aos critérios, de modo que estes pesos, possam representar sua importância (HORA, COSTA, 2015).

\section{PROPOSTA DE SOLUÇÃO}

\subsection{Critério Avaliados}

$>$ Preço: Nesse ponto busca-se pesquisar os preços de mercado, e encontrar uma opção que possa estar entre as médias pesquisadas.

$>$ Qualidade: Nesse ponto busca-se medir, a qualidade dos produtos adquiridos, assim como sua resistência ao acomodar diversos produtos, como conseguir preservar os produtos em suas viagens até o ponto de entrega.

Tempo de entrega: Este critério busca avaliar o tempo em dias, em que a mercadoria é comprada e de fato chega ao Centro de Distribuição para o uso. 


\subsection{Pesquisa de Qualidade}

A Qualidade das caixas de papelão foi medida através de uma pesquisa feita com 20 colaboradores aleatórios, que trabalham diretamente na operação e fazem a manipulação das caixas e produtos. Todos os 20 foram submetidos à pesquisa apresentada na Tabela 1.

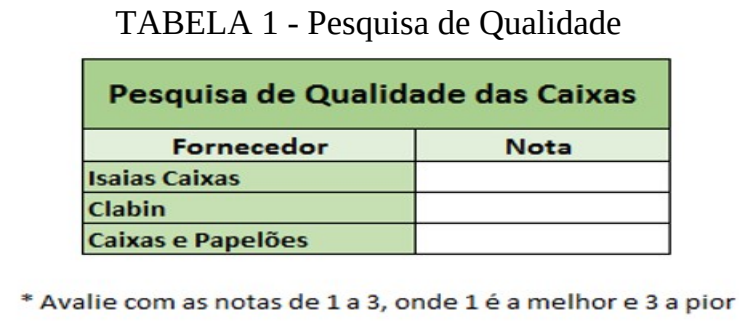

Fonte: Autores (2019)

Após a aplicação da pesquisa, obteve-se o resultado apresentado na Tabela 2.

TABELA 2 - Resultado da Pesquisa de Qualidade

\begin{tabular}{|c|c|c|c|c|}
\hline \multicolumn{4}{|c|}{ Pesquisa de Qualidade das Caixas } & \multirow{3}{*}{ 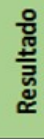 } \\
\hline \multirow{2}{*}{ Fornecedor } & \multicolumn{3}{|c|}{ Notas } & \\
\hline & Votos 1 & Votos 2 & Votos 3 & \\
\hline Isaias Caixas & 4 & 12 & 4 & $2^{\circ}$ \\
\hline Clabin & 12 & 5 & 3 & $1^{\circ}$ \\
\hline Caixas e Papelões & 4 & 3 & 13 & $3^{\circ}$ \\
\hline
\end{tabular}

Fonte: Autores (2019)

Observa-se que para os colaboradores que passaram pela pesquisa a caixa de melhor qualidade é aquela fornecida pela Clabin, seguida pelo fornecedor Isaias Caixas e por último as caixas fornecidas pela Caixas e Papelões. Com essas informações é possível fazer a medição de qualidade dos materiais através dos números de 1 a 3, onde 1 é ótimo e 3 ruim.

\subsection{Alternativas de Fornecedores}

Em meio a uma pesquisa de mercado, elencou-se 3 fornecedores que correspondem às exigências da empresa. A Tabela 3 mostra as informações do primeiro candidato a fornecedor "Isaías Caixas”. O fornecedor mostrou ter um preço acessível de mercado, com uma boa qualidade de materiais, tempo de entrega bom e todos os valores atendem as necessidades da empresa.

TABELA 3 - Informações de Isaias Caixas

\begin{tabular}{|l|c|}
\hline \multicolumn{2}{|c|}{ Isaias Caixas } \\
\hline Preço & $\mathrm{R} \$ 2,56$ \\
\hline Qualidade da Caixa & 2 \\
\hline Tempode Entrega & 2 dias \\
\hline
\end{tabular}

Fonte: Autores (2019)

A Tabela 4 mostra as informações do segundo candidato, a fornecedora "Clabin”. O segundo fornecedor, da mesma forma que o primeiro atendeu às necessidades pré-definidas pela empresa e foi selecionado como um possível fornecedor. O preço está dentro da média de mercado, a qualidade dos produtos é excelente, porém o tempo de entrega é alto em relação aos outros fornecedores. 
TABELA 4 - Informações de Clabin

\begin{tabular}{|l|c|}
\hline \multicolumn{2}{|c|}{ Clabin } \\
\hline Preço & $\mathrm{R} \$ 3,05$ \\
\hline Qualidade da Caixa & 1 \\
\hline Tempode Entrega & 7 dias \\
\hline
\end{tabular}

Fonte: Autores (2019)

A Tabela 5 mostra as informações do terceiro candidato a fornecedor, "Caixas e Papelões”. O terceiro fornecedor, também atendeu todas às especificações da empresa, assim como os demais elencados. O preço é alto em relação aos outros fornecedores selecionados, a qualidade está abaixo dos outros fornecedores, porém seu tempo de entrega é o melhor dentre todos os pesquisados.

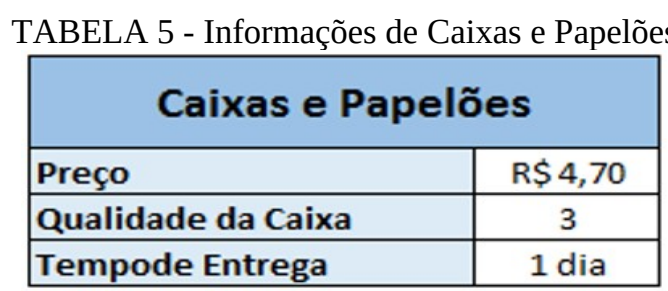

Fonte: Autores (2019)

\subsection{APLiCAÇÃo do MÉTOdo SAPEVo-M}

O método SAPEVO-M (Simple Aggregation of Preferences Expressed by Ordinal Vectors -Multi Decision Makers) representa uma nova versão do método ordinal original SAPEVO (Gomes et al. 1997) que possibilitava a avaliação de apenas um decisor. Esta versão evoluída estende o método a múltiplos decisores, além de introduzir um processo de normalização das matrizes de avaliação, incrementando a consistência do modelo (TEIXEIRA et al., 2019).

Segundo aqueles os mesmos autores, o método SAPEVO-M consiste, basicamente, em dois processos. Primeiramente, a transformação ordinal da preferência entre critérios, expressada por um vetor representando os pesos dos critérios. O segundo processo é a transformação ordinal da preferência entre alternativas dentro de um determinado conjunto de critérios. As informações de preferência no SAPEVO-M são denotadas por uma série de comparações pareadas entre as alternativas. A relação entre as alternativas é expressa em uma escala de sete pontos, na qual são mensuradas, relativamente, a importância entre as alternativas. A partir da avaliação entre alternativas, é obtida uma matriz com a representação numérica correspondente. $\mathrm{O}$ resultado da preferência entre as alternativas é expresso pelo vetor resultante da multiplicação matricial entre o vetor peso dos critérios $\mathrm{V}$ e a matriz de avaliação das alternativas $M$. As alternativas são, então, ordenadas em ordem decrescente dos valores numéricos obtidos, gerando-se o ranking desejado.

Para o desenvolvimento da pesquisa, foram selecionados 3 analistas de estoque, que são diretamente responsáveis por todo o setor e desempenham o papel de tomadores de decisões dentro do setor, que serão chamados de: Decisor 1, Decisor 2 e Decisor 3. Para começar a aplicação do método, é apresentada a Tabela 6, com a escala de critérios utilizada. 
TABELA 6 - Escala semântica do método SAPEVO

\begin{tabular}{|l|l|l|}
\hline Escala 1 (simbolo) & Escala 1 (variável/expressão Linguistica Correspondente) & Escala 2 \\
\hline$\ll \ll 1 ~$ & Absolutamente pior/Absolutamente menos importante & -3 \\
\hline$<\ll 1$ & Muito pior / Muito menos importante & -2 \\
\hline$\ll 1$ & Pior / Menos importante & -1 \\
\hline 1 & Igual ou equivalente / Tão importante quanto & 0 \\
\hline$» 1$ & Melhor / Mais importante & 1 \\
\hline$\gg » 1$ & Muito melhor / Muito mais importante & 2 \\
\hline$\gg \gg, 1$ & Absolutamente melhor / Absolutamente mais importante & 3 \\
\hline & Fonte: Teixeira, Santos e Gomes (2018) \\
\hline
\end{tabular}

Depois de conhecer os critérios a serem avaliados e a escala dos mesmos, os tomadores de decisão expressam suas preferencias, ordenando os critérios conforme suas opiniões e gerando para cada critério um determinado "peso", Tabela 7.

TABELA 7 - Vetor peso dos critérios.

\begin{tabular}{|c|c|c|c|c|}
\hline $\begin{array}{l}\text { DECISOR 1 } \\
\text { CRITÉRIO 1 } \\
\text { (PREÇO) }\end{array}$ & $\begin{array}{l}\text { DECISOR 2 } \\
\text { CRITÉRIO 1 } \\
\text { (PREÇO) }\end{array}$ & $\begin{array}{l}\text { DECISOR } 3 \\
\text { CRITÉRIO 1 } \\
\text { (PREÇO) }\end{array}$ & $\begin{array}{l}\text { PESO DO CRITÉRIO } 1 \\
\text { (SOMA) }\end{array}$ & $\begin{array}{c}\text { VETOR } \\
\text { NORMALIZADO } \\
\text { CRITÉRIO } 1\end{array}$ \\
\hline 1 & 1 & 1 & 3 & 3 \\
\hline $\begin{array}{c}\text { DECISOR 1 } \\
\text { CRITÉRIO 2 } \\
\text { (QUALIDADE) }\end{array}$ & $\begin{array}{c}\text { DECISOR 2 } \\
\text { CRITÉRIO 2 } \\
\text { (QUALIDADE) }\end{array}$ & $\begin{array}{c}\text { DECISOR } 3 \\
\text { CRITÉRIO 2 } \\
\text { (QUALIDADE) }\end{array}$ & $\begin{array}{l}\text { PESO DO CRITÉRIO } 2 \\
\text { (SOMA) }\end{array}$ & $\begin{array}{c}\text { VETOR } \\
\text { NORMALIZADO } \\
\text { CRITÉRIO 2 }\end{array}$ \\
\hline 0 & 0 & 0 & 0 & 0,011 \\
\hline $\begin{array}{c}\text { DECISOR 1 } \\
\text { CRITÉRIO } 3 \\
\text { (T. DE ENTREGA) }\end{array}$ & $\begin{array}{c}\text { DECISOR 2 } \\
\text { CRITÉRIO } 3 \\
\text { (T. DE ENTREGA) }\end{array}$ & $\begin{array}{c}\text { DECISOR } 3 \\
\text { CRITÉRIO } 3 \\
\text { (T. DE ENTREGA) }\end{array}$ & $\begin{array}{l}\text { PESO DO CRITÉRIO } 3 \\
\text { (SOMA) }\end{array}$ & $\begin{array}{c}\text { VETOR } \\
\text { NORMALIZADO } \\
\text { CRITÉRIO } 3 \\
\end{array}$ \\
\hline 0,285 & 0,333 & 0,5 & 1,118 & 1,118 \\
\hline
\end{tabular}

Fonte: Autores (2019).

Percebe-se que o maior peso foi dado ao preço, logo, ele pode ser considerado o critério mais importante para a escolha de um fornecedor. A qualidade das caixas ficou com o menor peso.

Cada avaliador realizou uma comparação paritária entre os fornecedores dentro de cada critério. A matriz de avaliação que reúne todos os dados dos critérios para cada fornecedor é apresentada na Figura 2.

\begin{tabular}{|c|c|c|c|c|}
\hline PREÇO & Decisor 1 & Decisor 2 & Decisor 3 & $\begin{array}{c}\text { Total ( somatório) } \\
\text { Preço }\end{array}$ \\
\hline Isaias Caixas & 1 & 1 & 1 & 3 \\
\hline Clabin & 0,666 & 0,333 & 0,714 & 1,713 \\
\hline Caixas e Papelões & 0 & 0 & 0 & 0 \\
\hline
\end{tabular}

\begin{tabular}{|c|c|c|c|c|}
\hline QUALIDADE & Decisor 1 & Decisor 2 & Decisor 3 & $\begin{array}{c}\text { Total ( somatório) } \\
\text { Qualidade }\end{array}$ \\
\hline Isaias Caixas & 0,714 & 0,5 & 0,8 & 2,014 \\
\hline Clabin & 1 & 1 & 1 & 3 \\
\hline Caixas e Papelões & 0 & 0 & 0 & 0 \\
\hline
\end{tabular}

\begin{tabular}{|c|c|c|c|c|}
\hline T. ENTREGA & Decisor 1 & Decisor 2 & Decisor 3 & $\begin{array}{c}\text { Total (somatório) } \\
\text { T. Entrega }\end{array}$ \\
\hline Isaias Caixas & 0,875 & 0,8 & 0,5 & 2,175 \\
\hline Clabin & 0 & 0 & 0 & 0 \\
\hline Caixas e Papelões & 1 & 1 & 1 & 3 \\
\hline
\end{tabular}

FIGURA 2 - Compilado a partir de informações dos tomadores de decisão. Fonte: Autores (2019) 
De acordo com os resultados obtidos, Tabela 8, observa-se que o fornecedor Isaias Caixas se destaca no critério preço, com uma pontuação muito superior aos outros, visto que o critério preço foi o determinado com maior peso. O fornecedor Clabin ganha um bom destaque na qualidade de caixas, porém o peso desse critério é inferior aos demais. O último fornecedor, Caixas e Papelões, teve grande destaque no tempo de entrega, vendo que o peso para esse critério também é alto.

TABELA 8 - Vetor peso dos critérios.

\begin{tabular}{|c|c|c|c|}
\hline FORNECEDOR & PREÇO & QUALIDADE & T. DE ENTREGA \\
\hline Isaias Caixas & 3 & 2,014 & 2,175 \\
\hline Clabin & 1,713 & 3 & 0 \\
\hline Caixas e Papelões & 0 & 0 & 3 \\
\hline
\end{tabular}

Fonte: Autores (2019)

\section{RESULTADOS ALCANÇADOS}

Observa-se que o resultado final, Tabela 9, gerou um grande destaque ao fornecedor Isaias Caixas, que saiu como a primeira e melhor opção como fornecedor, ao obter grande destaque no critério Preço, e se manter na média entre o tempo de entrega o permitiu ser selecionado como o melhor fornecedor para a empresa.

Em segundo lugar, a Clabin ficou muito abaixo da primeira opção, com menos da metade dos graus obtidos, mas se mostrou uma excelente opção de fornecedor, uma vez que seus preços são acessíveis e a qualidade dos seus produtos está acima da média de mercado.

Em terceiro lugar, como última opção, está a Caixas e Papelões, a empresa ganhou destaque no seu tempo de entrega onde, pode ficar muito acima dos outros fornecedores, porém, seus produtos eram os mais caros de todos os fornecedores elencados e a qualidade de seus produtos foram vistas como a pior.

TABELA 9 - Resultado ranqueamento.

\begin{tabular}{|c|c|c|}
\hline FORNECEDOR & GRAU OBTIDO & ORDENAÇÃO \\
\hline Isaias Caixas & 11,453 & $1^{\circ}$ \\
\hline Clabin & 5,172 & $2^{\circ}$ \\
\hline Caixas e Papelões & 3,354 & $3^{\circ}$ \\
\hline
\end{tabular}

Fonte: Autores (2019).

Todos os cálculos foram realizados por meio do aplicativo computacional SapevoWeb, disponível no site www.sapevoweb.com. O sistema SapevoWeb foi desenvolvido pelo Laboratório de Estudos de Governança, Gestão e Otimização (LEGGO), a partir de uma parceria entre o corpo técnico do Centro de Análise de Sistemas Navais (CASNAV), um grupo de pesquisa do Programa de Pós-Graduação em Engenharia de Produção da Universidade Federal Fluminense (UFF) e um grupo de pesquisa do Programa de Pós-Graduação em Engenharia de Sistemas e Computação do Instituto Militar de Engenharia (IME). 
Com os resultados obtidos, a lista para um possível fechamento de contrato de fornecedores de caixas de papelão é:

- $1^{\circ}$. Isaias Caixas

- $\quad 2^{\circ}$. Clabin

- $\quad 3^{\circ}$. Caixas e Papelões

\section{CONSIDERAÇÕES FINAIS}

O método SAPEVO-M mostrou-se adequado para o problema do presente estudo, apresentando um ranqueamento e solução para a escolha de um fornecedor adequado, com as características exigidas pela empresa. A partir da solução gerada pela aplicação do método foi possível para o tomador de decisão realizar uma escolha satisfatória.

\section{REFERÊNCIAS BIBLIOGRÁFICAS}

[1] ANDREOLI, T.; BASTOS, L. Gestão da Qualidade melhoria contínua e busca pela excelência. $1^{\text {a }}$ Ed. Curitiba. Paraná: Intersaberes, 2017.

[2] BRIOZO, R. A.; MUSETTI, M. A. Método multicritério de tomada de decisão: aplicação ao caso da localização espacial de uma Unidade de Pronto Atendimento UPA 24h. Gestão \& Produção, v. 22, n. 4, São Carlos, set. 2015, p. 805-8019.

[3] CARVALHO, M. A; Inovação em produtos. 2 ${ }^{a}$ Ed. São Paulo. Edgar Blucher Ltda, 2017.

[4] COSTA, C. C. M.; FERREIRA, M. A. M.; OLIVEIRA, L. G. Utilização do método multicritério no processo de tomada de decisão nas organizações rurais de pequeno porte. RACE, v. 12, n. 2, jul./dez. 2013, p. 491-524.

[5] GOMES, L. F. A. M., e GOMES, C. F. S. Princípios e métodos para a tomada de decisão: Enfoque multicritério (6a ed.). São Paulo: Atlas. (2019).

[6] GOMES, L. F. A. M., MURY, A. R., GOMES, C. F. S. (1997). Multicriteria Ranking with Ordinal Data. Systems Analysis Modelling Simulation, vol. 27, pp. 139 - 145.

[7] HORA, H. R. M.; COSTA, H. G. Proposta de um método multicritério para escolha múltipla. Production, v. 25, n. 2, São Paulo, abr./jun. 2015, p. 441-453.

[8] MOHR, J.; SENGUPTA, S.; SLATER, S. LUCHT, R. Marketing para mercados de alta tecnologia e de inovações. (J.G. Heloisa Coimbra, Trad) ed. São Paulo: Pearson, 2011.

[9] REIS, E.; LÖBLER, M. L. O processo decisório descrito pelo indivíduo e representado nos sistemas de apoio a decisão. RAC. v. 16, n. 3, Rio de Janeiro, Maio/Jun. 2012, p. 397-417.

[10] TEIXEIRA, L. F. H. S. B.; SANTOS, M.; GOMES, C. F. S. Proposta e implementação em python do método Simple Aggregation of Preferences Expressed by Ordinal Vectors - Multi Decision Makers: uma ferramenta web simples e intuitiva para Apoio à Decisão Multicritério. In: SIMPÓSIO DE PESQUISA OPERACIONAL E LOGÍSTICA DA MARINHA, 19., 2019, Rio de Janeiro, RJ. Anais [...]. Rio de Janeiro: Centro de Análises de Sistemas Navais, 2019. 
[11] TEIXEIRA, L. F. H. S. B.; SANTOS, M.; GOMES, C. F. S. SapevoWeb Software (v.1). 2018. Disponível em< $<$ http://www.sapevoweb.com> Acesso em 10/08/2019 SapevoWeb Software (v.1).

[12] WERNKE, R.; BORNIA, A. C. A contabilidade gerencial e os métodos multicriteriais. Revista Contabilidade \& Finanças. v. 12 n, 25, São Paulo, Jan./Abr. 2001, p. 60. 